\title{
Impact of Limited Memory Resources
}

\author{
Dave Binkley Dawn Lawrie Steve Maex Christopher Morrell \\ Loyola College \\ Baltimore MD \\ 21210-2699, USA \\ \{binkley,lawrie,smaex\}@cs.loyola.edu,chm@loyola.edu \\ Keywords: program comprehension, memory, identifier names
}

\begin{abstract}
Since early variable mnemonics were limited to as few as six to eight characters, many early programmers abbreviated concepts in their variable names. The past thirty years has seen a steady increase in permitted name length and, slowly, an increase in the actual length of identifiers. However, in theory names can be too long. Most obviously, in object-oriented programs, names often involve chaining of method calls and field selectors (e.g., class.firstAssignment().name.trim()). While longer names bring the potential for easier comprehension through more embedded sub-words, there are practical limits to length given limited human memory resources.

The central hypothesis studied herein is that names used in modern programs have reached this limit. Statistical models derived from an experiment involving 158 programmers of varying degrees of experience show that longer names extracted from production code take more time to process and reduce correctness in a simple recall activity. This has clear negative implications for any attempt to read, and hence comprehend or manipulate, the source code of modern software. The experiment also evaluates the advantage of identifiers having ties to a programmer's persistent memory. Combined these results reinforce past proposals advocating the use of limited, consistent, and regular vocabulary in identifier names. In particular, good naming limits length and reduces the need for specialized vocabulary.
\end{abstract}

\section{Introduction}

Early compilers limited identifier length starting a long tradition of abbreviation [8]. Modern programming languages have forgone such limits; thus, programmers have been encouraged to replace abbreviations, such as mnelmns, with ever longer names, such as max_num_elmns or even max_number_elements. However, a name can become too long. Consider trying to work with the_maximum_number_of_elements_seen_in_the_input even given an IDE that performs name completion.

The effective removal of length limitations provides programmers with considerable freedom to select names that promote source code understanding. With this freedom comes responsibility. For example, Liblit et al. observe that longer names, with more embedded sub-words, are more informative [8]. Furthermore, Lawrie et al. note that longer identifier names provide insight into the code's meaning [6, 7].

Yet, there is a practical limit to the length of identifiers, just as there is with the length of words in natural language. Jones observes that few developers appreciate how small short-term memory actually is. It only has the capacity to hold information on a few statements at most [4]. Thus, overloading a programmer's short-term memory is rather easy. One way of improving its capacity is to construct identifiers from subwords that include ties to the programmer's persistent memory.

This paper presents results from a study of the interplay between human memory limitations and entity names found within programs. The study is part of ongoing research into the impact that limited human memory resources have on program comprehension. While program comprehension clearly involves more than simple recall, such recall forms a fundamental and necessary precursor to essentially all comprehension activities. Thus, higher-level comprehension activites become impossible as a programmer's short-term memory becomes overcrowded, particularly when those activities require access to the crowded-out information.

In contrast to trends in modern programming language, the hypothesis that motivates this study is that identifiers can be too long negatively impacting recall and thus comprehension. While the existence of a theoretic limit is obvious, the study suggests that this limit has in fact been reached. The resulting degradation can be observed, for example, as a reduced ability to recall particular parts of the code caused by overcrowding of short-term memory. This is particularly 
an issue in object-oriented programming where 'chaining' often leads to long names created when several method calls and field selectors are concatenated together in a chain expression (e.g., name.trim().length()).

Maximal comprehension occurs when the pressure to create longer more expressive names is balanced against limited programmer short-term memory. Finding this balance point would provide guidance for creating easy-tocomprehend names. To this end, results from a study on the impact of name length and persistent-memory ties are presented. An important part of the study's construction is the use of production code as a source for the chain expressions (names). This means that the results are more apt to apply to existing rather than hypothetical programs. However, the study considers isolated names; therefore, it tends to underestimate the demand on memory because there is no need to remember neighboring code.

The remainder of the paper first presents some necessary background information in Section 2 before describing the experiment's design, the five hypotheses considered, and the results in Sections 3, 4, and 5, respectively. This is followed by a discussion of related work in Sections 6. Finally, Section 7 summarizes the paper and suggests some future work.

\section{Background}

This section describes background on memory, the findings from two prior motivating studies, and finally the statistical techniques used. Memory is the retention of information over time. It is broken down into three stages: sensory, short-term, and persistent. Sensory memory retains images, sounds, and smells for no more than a second. Interesting information is focused on and enters short-term memory. This second kind of memory is working storage. Short-term memory holds new information for about 15 to 30 seconds without rehearsal (repetition) [10]. The capacity of this subsystem is debatable, but limited. Miller first enumerated the capacity in the 1950s at seven plus or minus two items. However, more recent estimates indicate that it may be closer to three plus or minus one item [2]. One of the interesting aspects of short-term memory is that similar material can be combined into a chunk; this appears to increase the total amount of material remembered. Finally, persistent memory lasts from minutes to years. Persistent memory can be used to recall previously learned information including, for example, specific rules and repeated patterns such as those used in programming.

In the first of two prior studies, programmer comprehension was measured using three different levels of identifiers: short (often single letter), abbreviated, and full-word [6, 7]. Unexpectedly, programmers elicited essentially the same information from full-word and abbreviated identifiers.

The second prior study uses information from the English Lexicon Project. It showed that longer words required significantly greater time to determine their validity as actual words(greater lexical decision making time) [11]. One possible explanation for this phenomenon is that longer words require more fixations (landings of the eye) before they can be recognized and correctly classified.

Combined, the results of these two studies (the similar results for full words and abbreviations and the inhibitory influence of word length) suggest a study of the impact of limited short-term memory on source code comprehension. In other words, can names of the lengths used in modern programs be too long and thus crowd short-term memory? The study described herein presents statistical analyses that investigate this question.

As the statistical models are constructed from data that includes repeated-measures and missing values (e.g., due to participant drop out) linear mixed-effects regression models were used to analyze the data [13]. Such models easily accommodate unbalanced data, and, consequently, are ideal for this analysis. These statistical models allow the identification and examination of important explanatory variables associated with a given response variable.

The construction of a linear mixed-effects regression model starts with a collection of explanatory variables and may include a number of interaction terms. The interaction terms allow the effects of one explanatory variable on the response to differ depending upon the value of another explanatory variable. For example, if Java Experience interacts with Sex in a model where Correctness is the response variable, then the effect of Java Experience on Correctness depends on Sex (i.e., is different for men and women). Backward elimination of statistically non-significant terms $(p>0.05)$ yields the final model. Note that some non-significant variables and interactions are retained to preserve a hierarchically well-formulated model [9]. Therefore, individual $p$-values for terms participating in an interaction are not reported.

When interpreting the mixed-effects models described in Section 5, graphs are used to illustrate significant effects in the model. However, when the models have more than two explanatory variables it is not pragmatic to graph all the variables. Thus, when plots are constructed, variables not being discussed are set to their means or, in the case of categorical variables, a representative value.

\section{Experimental Design}

The study's design begins with a description of the source code selected, and then presents the layout of the experiment including the web-based applet used to collect the data, the explanatory and response variables collected, and the preparation of the raw data for analysis. The next section presents the five hypotheses formally studied using the collected data.

To investigate the interplay between memory and identifier selection, the study considers how well subjects retain 
the information contained in Java chain expressions. In doing so, it explores two orthogonal questions: what is the impact of the length of a chain expression on retention and what is the impact of familiarity with the parts (identifiers) that make up the chain expression.

\subsection{Source Code Selection}

The first step in constructing the study was to select the chain expressions (hereafter referred to simply as names as, taking a functional viewpoint, they name a resultant entity in a program). These expressions include both expressions denoting a method and its arguments. To balance the time required to take part in the study with the need to collect sufficient data from which to draw statistical conclusions, it was decided that eight questions would be included. The eight names used in the eight questions were extracted from production code. Choosing names from production code is important because it helps the experiment to more closely mirror an engineer's environment. However, selecting names from production code represents a compromise between control (e.g., the high-control of artificially constructed source [4]) and applicability of results to the code found in the real world.

The selected names were drawn from a collection of approximately 6.3 million lines of code taken from a cross section of open-source programs. In total 508,790 names were extracted. For each, the number of constituent parts (separated by Java's dot operator) was then computed. Figure 1 presents the distribution. For the experiment, those having only one or two parts were dropped because they were too short to create meaningful questions. The authors then scanned the remaining names and selected 200 representative examples. For each of these, the number of syllables was computed.

The eight names used in the study were then selected based on two criteria: length and ties. Length required having a similar number of parts and syllables. To simplify the initial data collection, the length was encoded as either short (having about 10 syllables and 4 parts) or long (having about 20 syllables and 7 parts). Ties further divided the names into those expected to be tied to persistent memory and those not expected to be tied to it. Here identifiers that appear in frequently used packages were assumed to have ties to persistent memory while program or even domain identifiers were not.

These two criteria divided the names into four categories: short with ties, short with no ties, long with ties, and long with no ties. To create a balanced study, two names from each category were selected. These are shown in Figure 2, where the underlined identifier is the part of the name to be recalled during the experiment.

\subsection{Experimental Layout}

The core of the experiment is laid out as eight questions. Each question is divided into three parts. The first shows

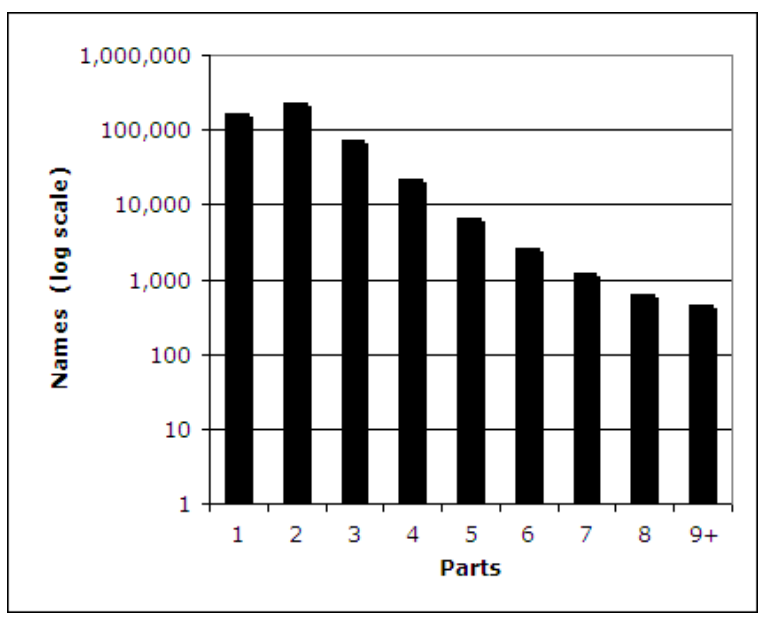

Figure 1. Extracted names categorized by number of parts (The $y$-axis uses a log scale).

a single name to the subject, who is free to study it for as long as desired. Then, as in similar studies, the subject undertakes a memory clearing activity. In this experiment subjects were asked to enter the kind of application from which they thought the name might have come. Finally, the subject is shown the name with one of the identifiers replaced by a blank and asked to enter the missing identifier.

The names used in the eight questions, shown in Figure 2, were arranged into two groups (labeled "Group 1" and "Group 2" in the figure). The questions within each group were shown in a random order to avoid systematic learning biases.

The experiment was conducted over the internet using a Java applet, which has several advantages. First, it allows for wide, quick distribution. Second, the applet prevents the use of the web browser's back button and thus provides flow control. Third, the applet allows timing how long subjects spend on each question. Finally, the results gathered are already in a digital format, which supports easy manipulation and statistical analysis and prevents data entry errors.

Before the eight questions, demographic data was collected using the screen shown in Figure 3. Figure 4 shows one set of the three screens used for each question. A final screen allows participants to provide comments and feedback on the study.

Subjects were recruited via email. Email was sent to current students, alumni of several colleges, and various professional groups, which described the study and included the URL where the applet was posted.

\subsection{Variables}

The variables collected during the experiment include two response variables and eleven explanatory variables. The first response variable, Time, represents the time spent examining the name shown on the first screen of each question. The second, Correctness, reflects the average judge- 
Short

Ties

(Group 1) Thread.currentThread().getName().substring(3)

From jakarta-jmeter/src/functions/org/apache/jmeter/functions/ThreadNumber.java

(Group 2) request.getQueryString().trim().length()

From jakarta-tomcat/src/share/org/apache/tomcat/core/RequestDispatcherlmpl.java

No Ties

(Group 1) digester.log.isDebugEnabled()

From jakarta-tomcat-connectors/util/java/org/apache/tomcat/util/digester/CallParamRule.java

(Group 2) payment.getJournalEntry().getTransactions()

From jaccounting-cvs-090905/.../src/java/com/spaceprogram/accounting/model/RecurrencePage.java

Long

Ties

(Group 1) fFriendViewer.setSelection(Math.min(index, fFriendViewer.getltemCount() - 1))

From eclipse/pde/internal/ui/editor/plugin/ExportPackageVisibilitySection.java

(Group 2) context.getDocumentBase().getProtocol().equalslgnoreCase(Constants.Request.WAR)

From jakarta-tomcat/src/share/org/apache/tomcat/core/DefaultServlet.java

No Ties

(Group 1) manager.getContainer().getLogger().debug(sm.getString(getStoreName())

From jakarta-tomcat-catalina/.../session/FileStore.java

(Group 2) paid.compareTo(invoice.getTotal().setScale(2, BigDecimal.ROUND_HALF_UP))

From jaccounting-cvs-090905/PaymentPage.java

Figure 2. The names used in the study's eight questions, categorized by length and the expectation that the missing identifier has ties to persistent memory. The missing identifier is underlined.

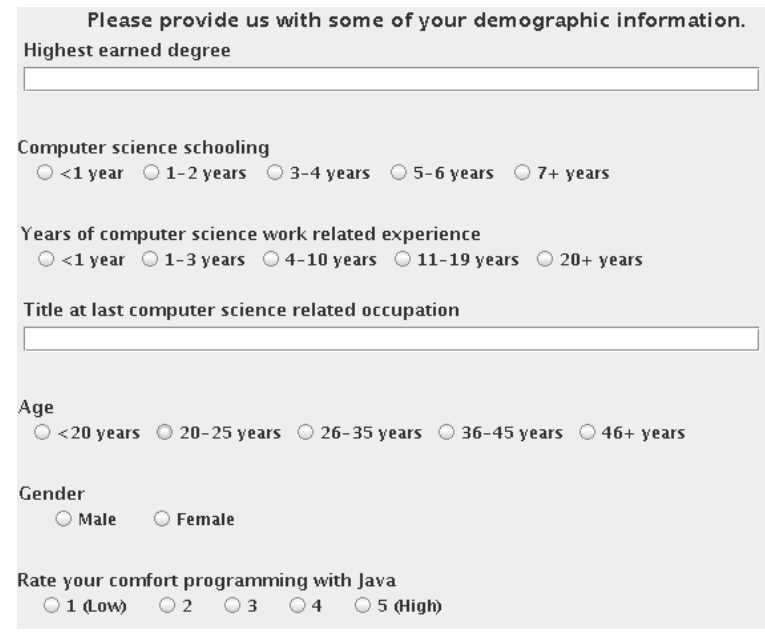

Figure 3. Demographics Screen

ment of the correctness of the answer on the third screen. Its computation is described below.

The explanatory variables come from two sources: the questions and the demographics. The four variables for each question include the Length (long or short) of the name, the Syllable Count, the number of Parts, and the existence of Ties to persistent memory. Length and Ties are described previously. The Syllable Count is the number of syllables in the name, and Parts is counted by separating the name based on Java dot-operators.

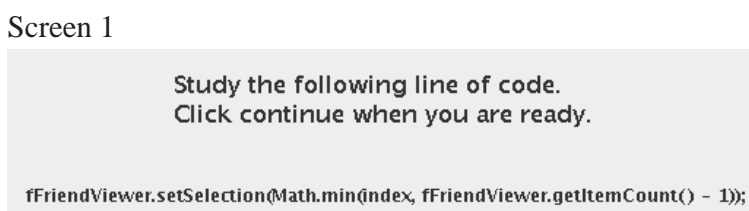

Screen 2

In a few words, describe the kind of application or the industry where this line of code might be found.

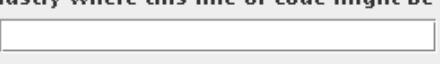

Screen 3

Fill in the blank to complete the previous line.

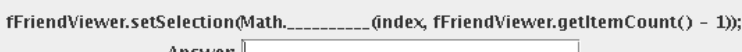

Answer: |

Figure 4. Screens of an example question

The seven demographic variables are used to uncover patterns that arise from who the subjects are. They include the Highest Degree earned, years of CS Schooling, years of Work Experience, Job Title, Age, Sex, and Java Experience (referred to as comfort in Figure 3). Many were collected as categorical variables to eliminate encoding errors. For example, from Figure 3, numeric values were collected categorically as ranges, limiting free-form data entry problems (e.g., Age entered as "142" or "old”). 


\subsection{Data Preparation}

Following administration of the experiment, the collected data was prepared for statistical analysis. The processing included categorizing free-form answers, considering the demographic data and comments from the final screen, and removing outliers. Each of these procedures is now discussed in more detail.

Three of the four free-form responses were encoded as categorical variables. The exception was the response to the memory clearing exercise. The first of the three, Highest Degree was categorized using the North American degree titles high school, bachelors, masters, MBA, and PhD. This required mapping some degrees from non-North American participants. Second, the title of the last computer science job was categorized as student, researcher, teacher, programmer, or other (e.g., professor was considered a researcher, lecturer a teacher, and architect a programmer).

The final free-form response was the value entered for the missing identifier. The result was scored on a scale from 0 to 4 . Exactly correct answers (sans case) were scored a 4 and almost correct answers were given a 3 . The score 2 was given to answers which had something right in them, and 1 to answers that were wrong. Zero was used for answers that were blank (e.g., caused by an errant double click) or where the subject wrote something to the effect that they got distracted. These entries were removed as they represent erroneous conditions.

Scoring was performed separately by two of the authors, and the results averaged. The authors judged each distinct response, so that all responses that were exactly the same received the same score. The number of distinct responses per question averaged 28.25 with a minimum of 14 and a maximum 44 distinct responses per question. Statistically, the scores of the two judges were in almost perfect agreement $(\kappa=0.802)$.

Based on information on the final screen or in the demographics, the data for a few subjects was removed. For example, one subject reported writing down each name. A second subject reported being a biology faculty member with little computer science training.

Finally, the time spent examining Screen 1 was examined. It was decided that responses with times shorter than 1.5 seconds should be removed because that gave the subject insufficient time to process the code. This effected 18 responses. Also excessively large values were removed. This effected 6 responses each longer than 9 minutes.

\section{Experimental Hypotheses}

This section presents the five hypotheses studied. Each hypothesis is given an informal name, which is followed by its formal statement and then its motivation. The next section presents the statistical analyses used to investigate each of the five hypotheses.
Hypothesis 1 Length increases study time

$\mathrm{H} 1_{0}$ : Study Time is the same regardless of Length

$\mathrm{H} 1_{A}$ : Length increases study Time

The first hypothesis states that longer names require more Time to consider, process, and, thus, understand. All other things being equal, increased Time equates to increased software cost (in particular, software maintenance cost). By itself, this result might not seem a great surprise, and the cost might be paid for through, for example, improved comprehension, fewer defects, or more efficient changes. However, if all things are not equal, for example if increased Length also increases other costs, then names can be too long.

\section{Hypothesis 2 Length reduces correctness \\ $\mathrm{H} 2_{0}$ : Correctness is the same regardless of Length $\mathrm{H} 2_{A}$ : Length reduces Correctness}

The second hypothesis states that longer names reduce subjects' ability to recall parts of a name. This is due to the over-crowding of short-term memory; thus, subjects are less able to recall particular parts of the name. An affirmative finding for both Hypotheses 1 and 2 would support the notion that names can be too long.

\section{Hypothesis 3 Memory ties improve correctness $\mathrm{H} 3_{0}$ : Ties do not effect Correctness $\mathrm{H} 3_{A}$ : Ties improve Correctness}

The third hypothesis states that common programming idioms are easier to recall and process because they have Ties to one or more concepts stored in the programmer's persistent memory. Thus, problems that involve more common Java expressions will have greater Correctness [4].

\section{Hypothesis 4 Experience improves correctness \\ $\mathrm{H} 4_{0}$ : Java Experience has no effect on Correctness \\ $\mathrm{H} 4_{A}$ : Java Experience improves Correctness}

The fourth hypothesis essentially reinforces Hypothesis 3 as greater experience is expected to lead to greater persistent memory Ties. Thus, together Hypotheses 3 and 4 support the notion that Java Experience improves Ties to persistent memory that can be exploited in code comprehension and, in this case, lead to higher Correctness.

\footnotetext{
Hypothesis 5 The effect of ties to persistent memory, length, and experience on correctness are interdependent

$\mathrm{H} 5_{0}$ : The effect of Ties, Length, and Java Experience on Correctness are independent

$\mathrm{H} 5_{A}$ : The effect of Ties, Length, and Java Experience on Correctness are interdependent
} 
Hypothesis 5 is interested in investigating whether or not Ties to persistent memory, Length, and Java Experience interact with each other. To obtain a clearer picture, this hypothesis is separated into three sub-Hypotheses. In each case the hypothesis states the interaction in its 'natural order'; however, each is in fact symmetric. In other words if the impact of $a$ increases (or decreases) with $b$ then the impact of $b$ increases (or decreases) with $a$.

\section{Hypothesis 5a The impact of memory ties on} correctness increases with length

H5 $\mathrm{a}_{0}$ : The impact of Ties to persistent memory on Correctness is independent of Length

H5 $\mathrm{a}_{A}$ : The impact of Ties to persistent memory on Correctness increases with Length

\section{Hypothesis 5b The impact of experience on correctness increases with ties \\ $\mathrm{H}_{5} \mathrm{~b}_{0}$ : Java Experience has no effect on the impact of persistent memory Ties on Correctness \\ $\mathrm{H}_{5} \mathrm{~b}_{A}$ : Java Experience increases the impact of persistent memory Ties on Correctness}

\section{Hypothesis 5c The impact of length on correctness decreases with experience \\ $\mathrm{H} 5 \mathrm{c}_{0}$ : The impact of Length on Correctness is independent of Java Experience \\ $\mathrm{H} 5 \mathrm{c}_{A}$ : The impact of Length on Correctness decreases with Java Experience}

The first of the three sub-hypotheses, Hypothesis 5a, states that Ties to persistent memory becomes more valuable as short-term memory becomes more crowded. Alternatively, with shorter names, Ties to persistent memory have less value. Hypothesis $5 \mathrm{~b}$ states that Java Experience becomes more valuable in the presence of Ties to persistent memory. Finally, Hypothesis $5 \mathrm{c}$ states that Length becomes less influential with greater Java Experience.

Support for these hypotheses will be evident in the statistical significance of an interaction between the relevant pair of explanatory variables. In a model, this kind of interaction appears as a difference in slopes. For example, as is shown in Figure 5, if the null hypothesis for 5b were rejected, the lines for Ties and no Ties would have different slopes.

\section{Experimental Results}

This section first describes the subject demographics and then, in five subsections, it considers the statistical models generated to investigate the five hypotheses. This is followed by a summary of the results and finally threats to the validity of the experiment. The study included 158 subjects, most of whom (97\%) had earned a university degree. In terms of the years of CS Schooling, $7 \%$ had 1 to 2 years, $34 \%$ had 3 to 4 years, $24 \%$ had 5 to 6 years, and 35\% had 7

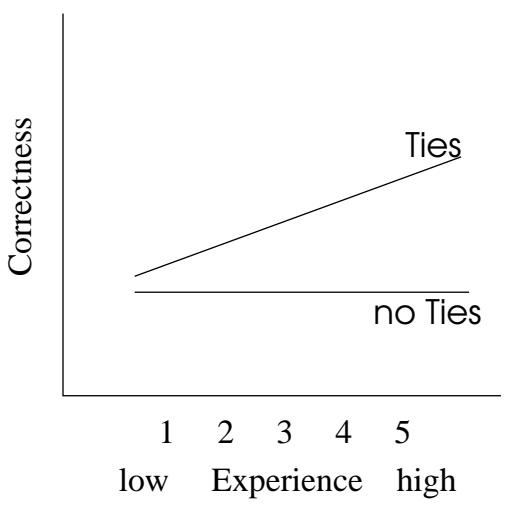

Figure 5. Hypothesis 5b expected output

or more years. The distribution of Age shows that a majority of subjects fell in the 26 to 35 age range (42\%) followed by the 20 to 25 age range (25\%). As would be expected, the years of Work Experience reflect Age with the peak being $34 \%$ falling into the 4 to 10 years category. The other groups were distributed between $13 \%$ and $20 \%$. The gender demographics ( $87 \%$ male) shows that the subject population mirrors the field in terms of men and women.

The study did draw more heavy from the research community than would be expected in a purely random sample with $34 \%$ researchers, $37 \%$ programmers, $15 \%$ other, $12 \%$ students, and 3\% teachers. This bias towards researchers can be expected to be associated with greater schooling. The statisical models show neither of these demographic variables as significant. Hence, neither effect is a significant concern. Finally, the distribution of the self-reported Java Experience was fairly uniform. This rating of subjects' comfort with Java was on a scale from 1 (low) to 5 (high) and breaks down from low to high as $16 \%, 14 \%$, $18 \%, 27 \%, 25 \%$; thus, just over half of the participants rated themselves above the average.

\subsection{Hypothesis 1 - Length increases study time}

The models considered for each hypothesis start with a collection of explanatory variables. Backwards elimination is then applied to iteratively remove those variables that are not statistically significant. When only a single explanatory variable is of interest, as is the case in the first model, then elimination will either remove the variable indicating no effect or it will retain it, indicating an effect.

To investigate the first hypothesis, the initial model includes the explanatory variable Length. This variable is significant $(p<0.0001)$, and indicates that longer names take an average of 20.1 seconds longer to process.

Because of this significance, an attempt to further understand this effect was undertaken. A second mixedeffects model was constructed, this time starting with the explanatory variables Syllable Count and Parts in place of Length. In this model, Parts is eliminated; thus, the final model includes only Syllable Count. In this, model, it 
takes an average of 1.80 seconds longer $(p<0.0001)$ for participants to process each additional syllable.

Both models, in particular, the more detailed second model, support the alternative hypothesis that as the length of a name increases, the Time required to process the name also increases.

\subsection{Hypothesis 2 - Length reduces correctness}

Similar to the first hypothesis, the initial model for Hypothesis 2 includes the explanatory variable Length. However, this variable is not significant $(p=0.778)$. To investigate possible reasons for this unexpected result and to parallel the analysis of Hypothesis 1, a second model was constructed beginning with the explanatory variables Syllable Count and Parts. Here both variables are significant: Correctness decreases by 0.0315 with each additional syllable ( $p=0.0019)$ and increases by 0.0768 for each additional part $(p=0.0174)$.

The interpretation of this second model is complicated by two factors. First, the coefficients of the two variables are opposite in sign (but when tested for, there was no interaction). This, in part, explains Length not being significant. The second complicating factor is that Parts and Syllable Count are (unsurprisingly) highly correlated ( $r=0.859$ ); thus, names with more Parts also tend to have more syllables. Regarding the first factor, it is relevant to note that the interpretation of coefficients in a statistical model is meant to be done "while holding other variables constant". However, in this case it is not practical to talk about varying Syllable Count while holding Parts constant (or visa versa) as the two are so strongly correlated.

Because the model indicates that both factors together are important in predicting Correctness two further models, which consider each of the variables independently, were constructed. Interestingly, both variables' significance decreases ( $p=0.0362$ for Syllable Count alone and $p=0.5582$ for Parts alone). For Syllable Count alone Correctness decreases by 0.0109 with each additional syllable. For Parts the slope is negative, as initially expected, but not significant.

Comparing the coefficient of Syllable Count in the two models where it appears, is instructive in gaining an understanding of the impact that the two variables have. In the model containing only Syllable Count, the coefficient of Syllable Count is less than in the joint model where Parts helps to dampen the (over) prediction of Syllable Count alone. The combination produces a better prediction.

Although more complex than the models for Hypothesis 1, the Hypothesis 2 models involving Syllable Count support the alternative hypothesis that Length reduces Correctness (recall). Combined with the first result, longer names are more costly as they take more time to process and lead to lower recall Correctness.

\subsection{Hypothesis 3 - Memory ties improve correctness}

The initial model for Hypothesis 3 includes the explanatory variable Ties, which is significant. In this model, Ties to persistent memory increase Correctness by 0.189 $(p=0.0009)$. Thus, there is statistical support to reject the null hypothesis and conclude that Ties to persistent memory improve recall Correctness.

\subsection{Hypothesis 4 - Experience improves correctness}

The initial model for Hypothesis 4 includes the explanatory variable Java Experience, which is significant. In this case, Correctness increases by $0.0849(p=0.0156)$ with each unit of Java Experience. Thus, there is statistical support to reject the null hypothesis and conclude that experience improves recall Correctness.

5.5 Hypothesis 5 - The effect of ties to persistent memory, length, and experience on correctness are interdependent

$$
\begin{aligned}
& 5 \mathrm{a}-\text { The impact of memory ties on correctness } \\
& \text { increases with length } \\
& 5 \mathrm{~b}-\text { The impact of experience on correctness } \\
& \text { increases with ties } \\
& 5 \mathrm{c}-\text { The impact of length on correctness decreases } \\
& \text { with experience }
\end{aligned}
$$

To test Hypothesis 5, the initial model includes the explanatory variables Length, Java Experience, and Ties, and all three pairwise interaction: Ties * Length, Java Experience * Ties, and Length * Java Experience. After the removal of non-significant terms, the final model includes the three explanatory variables and the interaction Length * Java Experience.

In this model there are two effects on Correctness. First, Ties to persistent memory brings an increase of 0.189 to Correctness ( $p=0.0009$ ). This means that, independent of other factors, subjects did better with more common Java names. Second, Length and Java Experience take part in an interaction ( $p=0.0336$ ) (thus their impact on the model cannot be stated independently).

This means that statistically the null hypothesis cannot be rejected for Hypotheses 5a nor 5b; however, for Hypothesis $5 \mathrm{c}$, the null hypothesis can be rejected. The interaction Length * Java Experience is shown in the top of Figure 6 by the different slopes of the two lines. Here, Java Experience has a greater impact when Length has the value long (the line with the greater slope). Symmetrically, to see that the impact of Length decreases with Java Experience observe that the gap between the two lines reduces with increased Java Experience. Interestingly, for those with the most experience, there is no difference in Correctness between short and long names.

Following the example used in previous models, the significance of Length, led to the the replacement of Length with Syllable Count and Parts in the generation of a new 

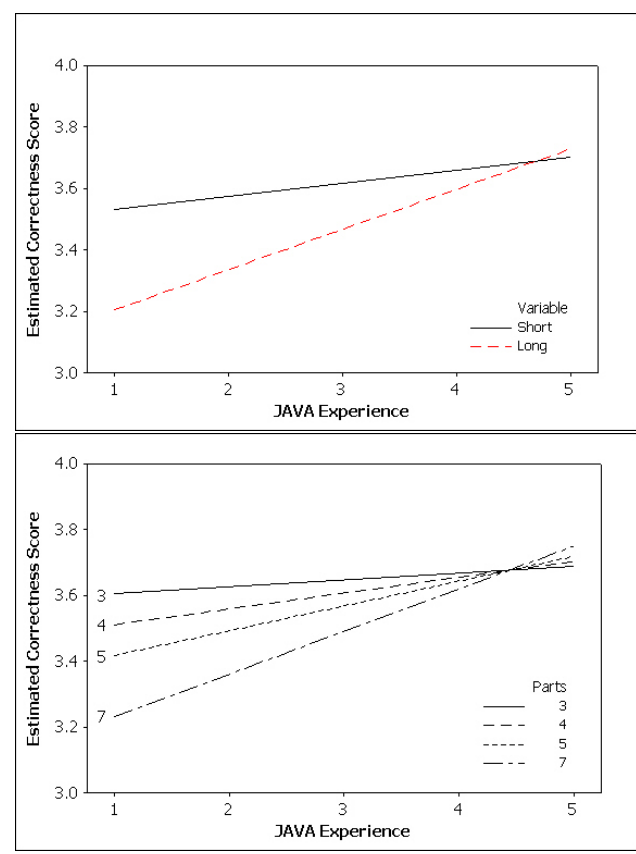

Figure 6. Interactions between Length and Java Exp. and between Parts and Java Exp..

model. The final model includes Java Experience, Ties, Parts, and Parts * Java Experience.

Again there are two effects on Correctness. First, Ties to persistent memory bring an increase of 0.209 to Correctness $(p=0.0004)$. The consistency of the occurrence and coefficient for Ties is indicative of a variable with a strong independent effect. Second, Parts and Java Experience take part in an interaction $(p=0.0249)$.

This again means that statistically the null hypothesis can be rejected for only Hypothesis 5c. The interaction Parts * Java Experience is shown in the bottom of Figure 6 by the four lines all having different slope (no 6-part names were used in the study). Comparing the two graphs in Figure 6, the greater detail provided when using Parts is visually evident. Similar to the Length model, Java Experience has a greater impact as the number of Parts increases (appearing as lines with greater slope). Symmetrically, for those with low Java Experience, Length has a greater impact.

Unlike Ties and Length, Java Experience is potentially influenced by subject demographics. Thus, a third model was constructed by adding to the prior model the (remaining) demographic variables: Highest Degree, CS Schooling, Work Experience, Job Title, Age, and Sex. This allows the model to determine if more general background or computer science experience plays a role in Correctness. The final model includes Java Experience, Ties, Parts, and Parts * Java Experience, just as before, with the same $p$-values and interpretation. This means that the effects measured in the study are not influenced by the additional demographic factors.
In summary, the interactions Length * Java Experience and Parts * Java Experience provide statistical evidence to reject the null hypothesis of Hypothesis 5c, and conclude that Java Experience's value increases with Length. It is not possible to reject the other two null hypotheses. In short, Ties appears to be of uniform value to all participants.

\subsection{Summary of Results}

Combined, these results reinforce past proposals advocating the use of limited, consistent, and regular vocabulary in identifier names. From Hypotheses 1 and 2, 'excess' length negatively impacts time and recall correctness. Furthermore, care in choosing a consistent vocabulary and in particular not to needlessly add to the vocabulary, is emphasized by the support for Hypotheses 3 and 4. Together, good naming principles limit length and reduce the need for specialized vocabulary. Finally, the support for Hypotheses 1, 2 , and $5 \mathrm{c}$ indicates that to a limited extent programmers can be trained to handle length related issues; however, this inevitably requires mental resources that might be better spent on other activities.

\subsection{Threats to validity}

There are four threats relevant to this research: external validity, internal validity, construct validity, and statistical conclusion validity. External validity, sometimes referred to as selection validity, is the degree to which the findings can be generalized to other (external) settings. In this experiment, selection bias is possible in the selected names; thus, results from the experiment may not be applicable to other source code. Careful selection of code from a large code base mitigates the impact of any such bias. It is also possible that taking the code out of context changes the recall process and thus the results when reading code in context would differ. Finally, selection bias is also possible in the selection of participants, which is clearly a convenience sample. The models involving the demographic data indicate that there is reasonable diversity in this population. Furthermore, the demographic variables were not statistically significant in any of the models.

Second, two threats to internal validity, the degree to which conclusions can be drawn about the causal effect of the explanatory variable on the response variables, exist. First, statistical associations do not imply causation; though, given the experimental setup, one should be able to infer that differences in performance are due to the explanatory variables considered. Second, learning effects occur when subjects believe that they have detected a pattern in the problems and attempt to exploit it to improve their performance on later problems. Questions were shown using a random order to avoid any systematic learning bias. Furthermore, the independence of the questions reduces the ability of subject to learn from past problems. Finally, other 
potential threats to internal validity, for example, history effects, attrition, and subject maturation [1] are non-issues given the short duration of the experiment.

Construct validity assesses the degree to which the variables used in the study accurately measure the concepts they purport to measure. Most of the study variables (e.g., Length and Age) can be measured precisely. This threat is only a concern for the variable Ties where the authors assessment of which identifiers are tied to persistent memory might differ form those with actual Ties.

Finally, a threat to statistical conclusion validity arises when inappropriate statistical tests are used or when violations of statistical assumptions occur. The models applied are appropriate for analyzing unbalanced, repeatedmeasures data, so that the conclusions drawn from the statistics should be valid.

\section{Related Work}

Related work comes on two 'sides' of this study: at a 'lower' level there is research on human memory and cognition and at a 'higher' level there is research on the impact of naming on programmer comprehension. To begin with, New et al. study the impact of word length on lexical decision latencies [11]. The study considered words that ranged from 3 letters to 13 letters, which, unlike its predecessors, represents the entire range of normally encountered English word lengths. They found that the number of syllables, letters, and, orthographic neighbors, each made significant independent contribution toward lexical decision latencies. For example, each syllable added 20 milliseconds to the time needed to recognize a word.

In terms of letters, the analysis revealed an unexpected U-shaped curve - decision latencies were greater for short and long words: word length was facilitatory from 3-5 letters, null from 5-8 letters, and inhibitory from 8-13 letters. One explanation offered for this phenomenon is that the reading of words with a length of 6-9 letters has the highest chances of being done in a single fixation. Shorter words are often skipped and longer words must be re-fixated as letters are more difficult to perceive the farther they are from the fixation point.

In terms of lexical decision latencies, most Java names are quite long because camel casing provides insufficient separation (e.g., the identifier EqualslgnoreCase is read as a sixteen letter word). Thus, they are well into the inhibitory range in terms of decision latency. This result underlies the study presented herein. It provides a general understanding of human lexical processing built upon in this study with a focus on processing in computer science.

The second related experiment considers the consequences of limited short-term memory's capacity on subjects' performance to comprehend short artificial code sequences [4]. This study provides results as to the impact that different character sequences have on the cognitive resources required during program comprehension. Two identifier attributes were studied: the amount of short-term memory required to hold an identifier's spoken form (i.e., the number of syllables) and pre-existence (i.e., ties to persistent memory). Thus, identifiers belonged to one of four possible sets: a single character whose spoken form contained a single syllable (short, no ties), an English word whose spoken form contained one syllable (short, ties), three characters not forming a word whose spoken form contained three syllables (long, no ties), and a word whose spoken form contained three syllables (long, ties).

In terms of correct answers, one syllable words received the most correct answers. This corresponds to short names with ties to persistent memory. The worst results were for three unrelated letters, which corresponds to long names with no ties to persistent memory and provides evidence of the crowding effect. In comparison, the study laid out in Section 3, is less artificial in that the code came from production programs rather than being constructed to satisfy experimental requirements. In exchange, the experimental conditions are not as tightly controlled. For example, the number of syllables for long names varied from 19 to 21 . This makes the results harder to analyze, but places the result closer to the experience of programmers.

The third study considers the work of Liblit et al., which aims to relate standard programming practice to modern theories of human language and cognition [8]. The authors find that "programmers use names in regular, systematic ways that reflect deep cognitive and linguistic influences. This allows names to carry [natural language] semantic cues that aid in program understanding and support software development. However, overuse of abbreviations can lead to a preponderance of unique symbols programmers must decipher [5], which may lead to inhibited understanding [8].

Liblit et al. observe, but do not study, "that if names are informative, then longer names, with more embedded subwords, should be more informative. Yet there are practical limits to the lengths of names, just as there are practical limits to the lengths of words in natural language. Longer names may be more informative, but they are also more cumbersome to read." As an example, they present two snippets that calculate Euclidean distance. In one identifiers have names such as distance_between_abscissae and first_ordinate, and in the others, identifiers have the more traditional names such as $\mathrm{dx}$ and $\mathrm{y} 1$. The longer names are more informative. In contrast, the length of the identifiers requires multiple fixations and more quickly overcrowds short-term memory; thus, requiring re-fixating and greater concentration. For a reader familiar with the abbreviations, the shorter names are easier to process. However, imagine reading the traditional names without previously knowing the Euclidean distance formula. Most programmers can recall this kind of situation in which a frag- 
ment of code with meaningless names was hard to comprehend until the semantics of the names became known.

Liblit et al. conclude that terse and abbreviated names enhance readability at the expense of expressiveness. The results from Section 5 dampen the expressiveness advantage by studying the memory demand of longer names. Similar to lexical decision latency studied by New et al., this suggests a U-shaped comprehension result in which names that are too short inhibit comprehension by failing to be sufficiently expressive, while those that are too long inhibit comprehension by overtaxing programmer short-term memory; thus, the results from Section 5 combined with those of Liblit et al. extend the results of New et al. to software.

Other researchers have studied naming's impact on comprehension at a 'higher' level. For example, Takang et al. investigate the hypothesis that programs with full-word identifiers are more understandable than those with abbreviations [12]. While a surprise to the authors, this hypothesis is not supported by the quantitative data collected. The study from Section 5 may shed some light on this unexpected result as the longer names may have overcrowded subject's short-term memory.

Finally, Deissenböck and Pizka present a formal model based on bijective mappings between concepts and names for adequate identifier naming [3]. The model is based on the observation that "research on the cognitive processes of language and text understanding shows that it is the semantics inherent to words that determine the comprehension process." The core idea is that within a given program a concept should always be referred to by the same name. Their goal is that names contain enough information for an engineer to comprehend the precise concept involved without too large a strain on short-term memory.

\section{Summary and Future Challenges}

The study described in this paper shows that names used in existing production code are long enough to crowd programmer's short-term memory. This provides evidence that software engineers need to consider shorter, more concise names [3]. The study extends prior results [4] from a highly controlled setting to a less controlled setting that, by using production code, more accurately mirrors real life.

The next step is to consider even less controlled situation and hence more realistic settings. For example, preliminary data for such a task was recently collected. The task involved debugging one of three versions of a function. The versions differ only in the length of their identifiers. Two trends appear in the initial data. First, increased length took increased time. Thus, there appears to be support for the notion that when debugging names can be too long. The second, and far more interesting observation comes from the trend between the abbreviated names and those constructed from full words. Here there appears to be an interaction between length and experience with length aiding experienced programmers while hurting inexperienced programmers. Further study of this affect is warranted.

\section{Acknowledgments}

Thanks to John Blair and Matt Hearn for preparation help. Special thanks to all the participants as this work would not be possible without their time. Finally, thanks to the anonymous reviewers for their careful reading of the experiment and suggestions for improvements to this paper and for future experiments to consider. This work is supported by NSF grant CCR-0305330.

\section{References}

[1] D. Sjøberg, J. Hannay, O. Hansen, V. Kampenes, A. Karahasanovic, N. Liborg, and A. Rekdal. A survey of controlled experiments in software engineering. IEEE Transactions on Software Engineering, 19(4), 1993.

[2] N. Cowan. The magical number 4 in short-term memory: a reconsideration of mental storage capacity. Behavioral and Brain Sciences, 24(1), 2001.

[3] F. Deißenböck and M. Pizka. Concise and consistent naming. In Proceedings of the 13th International Workshop on Program Comprehension (IWPC 2005), St. Louis, MO, USA, May 2005.

[4] D. Jones. Memory for a short sequence of assignment statements. $C$ $V u, 16(6)$, December 2004.

[5] Kari Laitinen. Estimating understandability of software documents. SIGSOFT Software Engineering Notes, 21(4), 1996.

[6] D. Lawrie, C. Morrell, H. Feild, and D. Binkley. What's in a name? A study of identifiers. In 14th International Conference on Program Comprehension, 2006.

[7] D. Lawrie, C. Morrell, H. Feild, and D. Binkley. Effective identifier names for comprehension and memory. NASA Journal of Innovations in Systems and Software Engineering, 3(4), 2007.

[8] B. Liblit, A. Begel, and E. Sweetser. Cognitive perspectives on the role of naming in computer programs. In 8th Annual Psychology of Programming Workshop, Brighton, UK, September 2006.

[9] C. Morrell, J. Pearson, and L. Brant. Linear transformation of linear mixed effects models. The American Statistician, 51, 1997.

[10] J.S. Nevid. Psychology Concepts and Applications. Houghton Mifflin Company, Boston, MA, 2003.

[11] B. New, L. Ferrand, C. Pallier, and M. Brysbaert. Reexamining the word length effect in visual word recognition: New evidence from the English Lexicon Project. Psychonomic Bulletin \& Review, 13(1), 2006.

[12] A. Takang, P. Grubb, and R. Macredie. The effects of comments and identifier names on program comprehensibility: an experiential study. Journal of Program Languages, 4(3), 1996.

[13] G. Verbeke and G. Molenberghs. Linear mixed models for longitudinal data. Springer-Verlag, New York, second edition, 2001. 